\title{
Integration through transients for Brownian particles under steady shear
}

\author{
M Fuchs ${ }^{1}$ and M E Cates ${ }^{2}$ \\ ${ }^{1}$ Fachbereich Physik, Universität Konstanz, D-78457 Konstanz, Germany \\ ${ }^{2}$ School of Physics, JCMB Kings Buildings, The University of Edinburgh, Mayfield Road, \\ Edinburgh EH9 3JZ, UK
}

\begin{abstract}
Starting from the microscopic Smoluchowski equation for interacting Brownian particles under stationary shearing, exact expressions for shear-dependent steady-state averages, correlation and structure functions, and susceptibilities are obtained, which take the form of generalized Green-Kubo relations. They require integration of transient dynamics. Equations of motion with memory effects for transient density fluctuation functions are derived from the same microscopic starting point. We argue that the derived formal expressions provide useful starting points for approximations in order to describe the stationary non-equilibrium state of steadily sheared dense colloidal dispersions.
\end{abstract}

\section{Introduction}

Colloidal dispersions can be driven into stationary non-equilibrium states by shearing. Their properties are important for the handling of dense colloidal dispersions, but yet not well understood from fundamental starting points [1]. A prominent and universally observed effect is shear thinning, that the viscosity of the solution decreases by orders of magnitude upon increasing the shear rate. A widely used many-body model of colloidal particles under shear is provided by the Smoluchowski equation [2], a special form of a Fokker-Planck equation [3], which, however, has yielded to exact solution only at low particle densities [4]. There it exhibits weak shear thinning followed by shear thickening. The model supposes the existence of a given solvent velocity flow profile that depends linearly on distance along one direction, as has been observed in polydisperse dense fluid and glassy colloidal systems [5, 6], and thus it does not include changes of the solvent velocity field induced by the particle motion. (Nor does it allow for 'shear banding' or other symmetry breaking phenomena.) An additional simplification of the model can be performed when solvent-induced interactions ('hydrodynamic interactions')

Konstanzer Online-Publikations-System (KOPS)

URL: http://www.ub.uni-konstanz.de/kops/volltexte/2007/3820/

URN: http://nbn-resolving.de/urn:nbn:de:bsz:352-38208 
are neglected, so that the model effectively describes interacting Brownian particles in a constant shear flow. Gratifyingly, shear thinning has been observed in simulations of this system [7], where also homogeneous states in a linear flow profile were recorded. The model thus contains flow curves (namely curves of stress versus shear rate) in qualitative agreement with typical experimental data of systems close to glassy arrest [8] at not too high shear rates (where hydrodynamic interactions presumably dominate [1]).

Recently we presented a mode-coupling approach which leads to a consistent and (in principle) parameter-free, quantitative, albeit approximate description of the stationary sheared state at high particle concentrations or strong interactions [9]. It explains the behaviour of dense dispersions under shear from considering the competition of local caging of particles [10], which causes slow structural relaxations, with shear advection of fluctuations [11], which speeds up the decay. As an important concept it uses integration of the transient dynamics in order to gain insights into the stationary state presumed to be reached at late times. A number of rather universal predictions of the approach have already been obtained [12], and are in qualitative agreement [13] with, for example, the mentioned computer simulations [7]. In this contribution we present details of the approach, starting from the many-body Smoluchowski equation with shear and setting up the frame for integrating through the transient. Formally exact expressions for stationary averages, correlation functions and susceptibilities, and for transient density correlators are presented. These exact results lay the foundations for our approach to sheared colloids, whose approximations, outlined in [9], will be given in detail in a companion paper. In particular the results derived below create a framework within which to make mode-coupling-type approximations for sheared colloids, without invoking the equilibrium form of the fluctuation-dissipation theorem (which cannot reliably be used under shear).

It is well known in many physical situations that the same standard approximation (e.g., factorizing an average) gives different results when applied to two formulations of a problem that would, if treated exactly, be equivalent. A careful choice is then required, and the work reported here can be thought of as 'preparing the best ground' for a judicious mode-coupling approach to sheared colloids.

The derived generalized Green-Kubo relations and generalized Zwanzig-Mori equations, which may also be of interest on their own, are useful to describe the non-equilibrium steady state because they enable one to connect the stationary distribution function to the transient dynamics evaluated with equilibrium averaging. This strategy was followed in mode-coupling calculations of the nonlinear viscosity of simple liquids under shear, where the slow relaxation of 'long-time tails' leads to non-analytic dependences on the shear rate [14]. Transient fluctuation functions were also successfully used in some of the simulation studies of this problem, and the connection to the theoretical approach was shown explicitly [15]. Here shearing cuts off the anomalous long-time dynamics present in the quiescent fluids, and subtle (but small) corrections to the viscosity arise.

Recent mode-coupling theory approaches to simple liquids close to glassy arrest by Miyazaki and Reichman [16, 17], and to violations of the fluctuation-dissipation theorem in Brownian particle systems by Szamel [18], follow a somewhat different approach from ours. There, time-dependent correlation functions for fluctuations around the sheared steady state are obtained, as are susceptibilities describing the response of the state in [18]. Thus, in the spirit of the mode-coupling theory of quiescent systems [10], structural quantities of the stationary state, which now depend on shear rate, enter as input into the equations describing the dynamics. Importantly, Miyazaki and Reichman find that shear advection of density fluctuations speeds up the structural relaxation, which would become excessively slow close to glassy arrest. 
Our approach, as sketched in [9], uses generalized Green-Kubo relations to access the stationary distribution function, in order to allow for its (possibly) non-analytic dependence on shear rate. We expect non-analyticities to arise when we consider the rheological properties of the system close to solidification into a colloidal glass, because the quiescent dynamics becomes non-ergodic at a glass transition described by the idealized mode-coupling theory [10]. Therefore, in this contribution, (i) in section 3 stationary averages are reformulated so that the transient dynamics enters; in the companion paper (in preparation) we will present in detail mode-coupling approximations so that the transient dynamics is described by transient density fluctuation functions. Then (ii) in section 4 , the equations of motion of transient density functions are reformulated in such a way that dense systems can be described, where particle interactions lead to large memory effects. In section 2 the model is defined and some properties discussed, while section 5 concludes with a short outlook to the companion paper.

\section{Steady-state properties}

\subsection{Microscopic starting point}

The system considered consists of $N$ spherical particles (diameter $d$ ) dispersed in a volume $V$ of solvent with imposed flow profile $\mathbf{v}(\mathbf{r})=\kappa \cdot \mathbf{r}$, where for simple shear with velocity along the $x$-axis and its gradient along the $y$-axis, the shear rate tensor is $\kappa=\dot{\gamma} \hat{\mathbf{x}} \hat{\mathbf{y}}$ (namely $\kappa_{\alpha \beta}=\dot{\gamma} \delta_{\alpha x} \delta_{\beta y}$ ). The effect of the shear rate $\dot{\gamma}$ on the particle dynamics is measured by the Peclet number [1], $P e_{0}=\dot{\gamma} d^{2} / D_{0}$, formed with the (bare) diffusion coefficient $D_{0}$ of a single particle. Dimensionless units are obtained by setting $d=D_{0}=k_{\mathrm{B}} T=1$, whereupon $P e_{0}=\dot{\gamma}$. The evolution of the distribution function $\Psi(\Gamma)$ of the particle positions, $\mathbf{r}_{i}$, $i=1, \ldots, N$ (abbreviated into $\Gamma=\left\{\mathbf{r}_{i}\right\}$ ), under internal forces $\mathbf{F}_{i}=-\partial_{i} U(\Gamma)$ (with the total interaction potential $U$ ) and shearing, but neglecting hydrodynamic interactions, is given by the Smoluchowski equation $[1,2]$ :

$$
\begin{aligned}
& \partial_{t} \Psi(\Gamma, t)=\Omega(\Gamma) \Psi(\Gamma, t), \\
& \Omega=\Omega_{\mathrm{e}}+\delta \Omega=\sum_{i} \partial_{i} \cdot\left(\partial_{i}-\mathbf{F}_{i}-\kappa \cdot \mathbf{r}_{i}\right) .
\end{aligned}
$$

Here $\Omega_{\mathrm{e}}=\sum_{i} \partial_{i} \cdot\left(\partial_{i}-\mathbf{F}_{i}\right)$ abbreviates the Smoluchowski operator (SO) without shear. In the following, operators act on everything to the right, if not marked differently by bracketing. The conditional probability, for the system to evolve from state point $\Gamma^{\prime}$ at time $t^{\prime}$ to $\Gamma$ at the later time $t$, denoted by $P\left(\Gamma t \mid \Gamma^{\prime} t^{\prime}\right)$, is also determined from $\Omega$ :

$$
\partial_{t} P\left(\Gamma t \mid \Gamma^{\prime} t^{\prime}\right)=\Omega(\Gamma) P\left(\Gamma t \mid \Gamma^{\prime} t^{\prime}\right),
$$

with the initial condition $P\left(\Gamma t \mid \Gamma^{\prime} t\right)=\delta\left(\Gamma-\Gamma^{\prime}\right)$, that both state points coincide at the same time.

There exist two special time-independent distribution functions: the equilibrium one, $\Psi_{\mathrm{e}}$, and the stationary one, $\Psi_{\mathrm{s}}$, which satisfy

$$
\Omega_{\mathrm{e}} \Psi_{\mathrm{e}}=0, \quad \Omega \Psi_{\mathrm{s}}=0
$$

The equilibrium one is determined from the total internal interaction energy $U$ via the Boltzmann weight, $\Psi_{\mathrm{e}}(\Gamma) \propto \mathrm{e}^{-U(\Gamma)}$, but the stationary distribution function $\Psi_{\mathrm{s}}$ is unknown. Averages with $\Psi_{\mathrm{e}}$ will be abbreviated by $\langle\cdots\rangle=\int \mathrm{d} \Gamma \Psi_{\mathrm{e}}(\Gamma) \ldots$, while $\Psi_{\mathrm{s}}$ determines steadystate averages, denoted by $\langle\cdots\rangle^{(\dot{\gamma})}=\int \mathrm{d} \Gamma \Psi_{\mathrm{s}}(\Gamma) \ldots$

At finite shearing, steady-state averages $f$, time-dependent correlation $C_{f g}(t)$ and timeindependent structure functions $S_{f g}$, for fluctuations $\delta f=f-\langle f\rangle^{(\dot{\gamma})}$ around the steady state, 
and response susceptibilities $\chi_{f g}(t)$, are the central objects of interest:

$$
\begin{aligned}
f(\dot{\gamma}) & =\langle f\rangle^{(\dot{\gamma})}=\int \mathrm{d} \Gamma \Psi_{\mathrm{s}}(\Gamma) f(\Gamma) \\
C_{f g}(t) & =\int \mathrm{d} \Gamma \int \mathrm{d} \Gamma^{\prime} W_{2}\left(\Gamma t+t^{\prime}, \Gamma^{\prime} t^{\prime}\right) \delta f^{*}\left(\Gamma^{\prime}\right) \delta g(\Gamma) \\
& =\left\langle\delta f^{*} \mathrm{e}^{\Omega^{\dagger} t} \delta g\right\rangle^{(\dot{\gamma})} \\
S_{f g}= & C_{f g}(t=0)=\left\langle\delta f^{*} \delta g\right\rangle^{(\dot{\gamma})} \\
\chi_{f g}(t) & =\left\langle\sum_{i} \frac{\partial f^{*}}{\partial \mathbf{r}_{i}} \cdot \partial_{i} \mathrm{e}^{\Omega^{\dagger} t} g\right\rangle^{(\dot{\gamma})} .
\end{aligned}
$$

The calculation of the fluctuation functions involves the joint probability distribution, $W_{2}\left(\Gamma t, \Gamma^{\prime} t^{\prime}\right)$, that the system is at point $(\Gamma, t)$ after it was in a stationary state at $\left(\Gamma^{\prime}, t^{\prime}\right)$; it is given using the conditional probability that is the solution of equation (2):

$$
W_{2}\left(\Gamma t, \Gamma^{\prime} t^{\prime}\right)=P\left(\Gamma t \mid \Gamma^{\prime} t^{\prime}\right) \Psi_{\mathrm{s}}\left(\Gamma^{\prime}\right)=\mathrm{e}^{\Omega(\Gamma)\left(t-t^{\prime}\right)} \delta\left(\Gamma-\Gamma^{\prime}\right) \Psi_{\mathrm{s}}\left(\Gamma^{\prime}\right) .
$$

Exchanging the order of times, $t^{\prime}>t$, it obeys $W_{2}\left(\Gamma t, \Gamma^{\prime} t^{\prime}\right)=W_{2}\left(\Gamma^{\prime} t^{\prime}, \Gamma t\right)$. The adjoint of the $\mathrm{SO}$ arose in the fluctuation function $C(t)$ from partial integrations:

$$
\Omega^{\dagger}=\sum_{i}\left(\partial_{i}+\mathbf{F}_{i}+\mathbf{r}_{i} \cdot \boldsymbol{\kappa}^{\mathrm{T}}\right) \cdot \partial_{i},
$$

where surface contributions are neglected, throughout, for the considered infinite system $(V \rightarrow \infty)$. The susceptibility $\chi_{f g}(t)$ describes the linear change of the expectation value of variable $g$ :

$$
\Delta g(\dot{\gamma})(t)=\langle g\rangle^{\left(\dot{\gamma}, h_{\mathrm{e}}\right)}-\langle g\rangle^{(\dot{\gamma})}=\int_{-\infty}^{t} \mathrm{~d} t^{\prime} \chi_{f g}\left(t-t^{\prime}\right) h_{\mathrm{e}}\left(t^{\prime}\right)+\mathcal{O}\left(h_{\mathrm{e}}^{2}\right),
$$

upon application of an external field $h_{\mathrm{e}}(t)$ that couples to the variable $f^{*}$ in the potential energy; namely, when the potential energy $U$ is perturbed to

$$
U \rightarrow U-f^{*}(\Gamma) h_{\mathrm{e}}(t) .
$$

The standard calculations [3] leading from equation (7) to equation (4) are sketched in the appendix.

Without applied shear the SO $\Omega_{\mathrm{e}}^{\dagger}$ is a Hermitian operator with respect to equilibrium averaging [2]:

$$
\left\langle\left(\Omega_{\mathrm{e}}^{\dagger} f^{*}\right) g\right\rangle=\left\langle f^{*} \Omega_{\mathrm{e}}^{\dagger} g\right\rangle=-\sum_{i}\left\langle\frac{\partial f^{*}}{\partial \mathbf{r}_{i}} \cdot \frac{\partial g}{\partial \mathbf{r}_{i}}\right\rangle,
$$

and (as is seen from specializing to $f=g$ ) it possesses a negative semi-definite spectrum. But with shear $\Omega^{\dagger}$ cannot be brought into a Hermitian form [3]; see section 2.2.1 below. The action of $\Omega$ on the equilibrium distribution function $\Omega \Psi_{\mathrm{e}}=\delta \Omega \Psi_{\mathrm{e}}$ will become important later on and allows one to define the stress tensor:

$$
\begin{aligned}
\delta \Omega \Psi_{\mathrm{e}} & =-\sum_{i} \partial_{i} \cdot \boldsymbol{\kappa} \cdot \mathbf{r}_{i} \Psi_{\mathrm{e}}=-\sum_{i}\left(\mathbf{F}_{i} \cdot \boldsymbol{\kappa} \cdot \mathbf{r}_{i}+\operatorname{Trace}\{\boldsymbol{\kappa}\}\right) \Psi_{\mathrm{e}} \\
& =\operatorname{Trace}\{\boldsymbol{\kappa} \sigma\} \Psi_{\mathrm{e}}=\dot{\gamma} \sigma_{x y} \Psi_{\mathrm{e}},
\end{aligned}
$$

with $\sigma_{\alpha \beta}$ the zero-wavevector limit of the potential part of the stress tensor:

$$
\sigma_{\alpha \beta}=-\sum_{i}\left(\delta_{\alpha \beta}+F_{i}^{\alpha} r_{i}^{\beta}\right)
$$

The specific form of $\kappa$ for sheared systems was used in the last equality of equation (9) only. 


\subsection{Basic properties}

Some well known properties of solutions of Fokker-Planck equations [3] shall be collected which bear relevance to the discussion of sheared colloidal dispersions.

2.2.1. Eigenfunctions expansions. The Smoluchowski equation of equation (1) may be viewed as a continuity equation in phase space [3], $\partial_{t} \Psi(\Gamma, t)+\sum_{i} \partial_{i} \cdot \mathbf{J}_{i}(\Gamma, t)=0$, where the probability current equals

$$
\mathbf{J}_{i}(\Gamma, t)=\left(\mathbf{F}_{i}+\kappa \cdot \mathbf{r}_{i}-\partial_{i}\right) \Psi(\Gamma, t) .
$$

Stationarity implies $\sum_{i} \partial_{i} \cdot \mathbf{J}_{i}(\Gamma, t \rightarrow \infty)=0$. But only if the current vanishes, $\mathbf{J}_{i}=0$, in the steady state, can one show that the SO $\Omega$ is related to an Hermitian operator. Necessary conditions for $\mathbf{J}_{i}=0$ are the 'potential conditions':

$$
\partial_{i}^{\alpha}\left(F_{j}^{\beta}+\left(\boldsymbol{\kappa} \cdot \mathbf{r}_{j}\right)^{\beta}\right)=\partial_{j}^{\beta}\left(F_{i}^{\alpha}+\left(\boldsymbol{\kappa} \cdot \mathbf{r}_{i}\right)^{\alpha}\right)
$$

While the potential conditions hold in equilibrium, they are violated under shear, because $\partial\left(\hat{\mathbf{y}} \cdot \boldsymbol{\kappa} \cdot \mathbf{r}_{i}\right) / \partial x_{i} \neq \partial\left(\hat{\mathbf{x}} \cdot \boldsymbol{\kappa} \cdot \mathbf{r}_{i}\right) / \partial y_{i}$. Then, if an expansion in eigenfunctions of the SO exists, it will have the following properties: the conditional probability from equation (2) takes the form

$$
P\left(\Gamma t \mid \Gamma^{\prime} t^{\prime}\right)=\sum_{n} \varphi_{n}(\Gamma) \hat{\varphi}_{n}\left(\Gamma^{\prime}\right) \mathrm{e}^{-\lambda_{n}\left(t-t^{\prime}\right)}
$$

where the eigenvalues satisfy $\operatorname{Re} \lambda_{n} \geqslant 0$, and the sets of eigenfunctions

$$
\Omega \varphi_{n}=-\lambda_{n} \varphi_{n} \quad \text { and } \quad \Omega^{\dagger} \hat{\varphi}_{n}=-\lambda^{*} \hat{\varphi}_{n},
$$

are bi-orthogonal, namely:

$$
\int \mathrm{d} \Gamma \hat{\varphi}_{n}(\Gamma) \varphi_{m}(\Gamma)=\delta_{n m}
$$

Yet, because no further connection between the sets of eigenfunctions exists in general, important properties of equilibrium fluctuations cannot be expected under shear: for example, the autocorrelation functions $C_{f f}(t)$ of equation (4) can fail to be of positive type and may exhibit negative frequency spectra [19].

2.2.2. Fluctuation-dissipation theorem. In the case without shear, where $\Omega_{\mathrm{e}}$ is the $\mathrm{SO}$ and averages are performed with the equilibrium distribution function $\Psi_{\mathrm{e}}$, a simple relation exists between the fluctuation function $C_{f g}^{(e)}(t)$ and the susceptibility $\chi_{f g}^{(e)}(t)$. By partial integration, and recalling that $\partial_{i} \Psi_{\mathrm{e}}=\mathbf{F}_{i} \Psi_{\mathrm{e}}$, one finds:

$$
\begin{aligned}
\chi_{f g}^{(e)}(t) & =-\left\langle\delta f^{*} \Omega_{\mathrm{e}}^{\dagger} \mathrm{e}^{\Omega_{\mathrm{e}}^{\dagger} t} \delta g\right\rangle \\
& =-\partial_{t} C_{f g}^{(e)}(t) .
\end{aligned}
$$

The expected fluctuation-dissipation theorem (FDT) connects the response and fluctuation function. On the other hand, with shear the susceptibility $\chi_{f g}(t)$ is connected to a fluctuation function of a variable $\tilde{f}$, which can only be found if the stationary distribution function is known [3, 18]; it satisfies

$$
\sum_{i} \partial_{i} \cdot \frac{\partial f^{*}}{\partial \mathbf{r}_{i}} \Psi_{\mathrm{s}}=\Omega \tilde{f}^{*} \Psi_{\mathrm{s}},
$$

and the FDT then states [3]:

$$
\chi_{f g}(t)=-\left\langle\delta \tilde{f}^{*} \Omega^{\dagger} \mathrm{e}^{\Omega^{\dagger} t} \delta g\right\rangle^{(\dot{\gamma})}=-\partial_{t} C_{\tilde{f} g}(t) .
$$

This appears not to be as useful as equation (16). 
2.2.3. Aspects of translational invariance. Homogeneous amorphous systems shall be studied so that by assumption the equilibrium distribution function $\Psi_{\mathrm{e}}$ is translationally invariant and isotropic. As shown in section 3.1, the steady-state distribution function with shear, $\Psi_{\mathrm{s}}$, is then also translationally invariant, assuming that no spontaneous symmetry breaking takes place, but anisotropic. Appreciable simplifications follow for steady-state quantities of wavevector-dependent fluctuations:

$$
f_{\mathbf{q}}(\Gamma, t)=\mathrm{e}^{\Omega^{\dagger} t} \sum_{i} X_{i}^{f}(\Gamma) \mathrm{e}^{\mathrm{i} \mathbf{q} \cdot \mathbf{r}_{i}},
$$

where, for example, $X_{i}^{\varrho}=1$ describes density fluctuations $\varrho_{\mathbf{q}}(t)$, while $X_{i}^{\sigma}=\delta_{\alpha \beta}+$ $(1 / 2) \sum_{j}^{\prime}\left(r_{i}^{\alpha}-r_{j}^{\alpha}\right) \mathrm{d} u\left(\left|\mathbf{r}_{i}-\mathbf{r}_{j}\right|\right) / \mathrm{d} r_{i}^{\beta}$ gives the stress tensor element $\sigma_{\alpha \beta}(\mathbf{q})$ for interactions described by the pair-potential $u$. Translational invariance in an infinite sheared system dictates that averages are independent of identical shifts of all particle positions, $\Gamma \rightarrow \Gamma^{\prime}$, where $\mathbf{r}_{i}^{\prime}=\mathbf{r}_{i}+\mathbf{a}$ for all $i$. Under such a shift the SO becomes

$$
\Omega^{\dagger}(\Gamma)=\Omega^{\dagger}\left(\Gamma^{\prime}\right)-\mathbf{a} \cdot \boldsymbol{\kappa}^{\mathrm{T}} \cdot \mathbf{P}, \quad \text { with } \mathbf{P}=\sum_{i} \partial_{i} .
$$

Thus a fluctuation of a variable which depends on particle separations only, namely $X_{i}^{f}(\Gamma)=$ $X_{i}^{f}\left(\Gamma^{\prime}\right)$ so that $\mathbf{P} X_{i}^{f}(\Gamma)=0$ holds, transforms to

$$
f_{\mathbf{q}}(\Gamma, t)=\mathrm{e}^{-\mathrm{i}(\mathbf{q}+\mathbf{q} \cdot \kappa t) \cdot \mathbf{a}} f_{\mathbf{q}}\left(\Gamma^{\prime}, t\right) .
$$

As the integral over phase space must agree for either integration variables $\Gamma$ or $\Gamma^{\prime}$, steadystate averages from equation (4) can be non-vanishing for zero wavevector only:

$$
f_{0}(\dot{\gamma}) \delta_{\mathbf{q}, \mathbf{0}}=\frac{1}{V}\left\langle f_{\mathbf{q}}(t)\right\rangle^{(\dot{\gamma})}
$$

In the following the index 0 will often be suppressed in, for example, the average density $\varrho=N / V$ and the shear stress $\sigma(\dot{\gamma})=\left\langle\sigma_{x y}\right\rangle^{(\dot{\gamma})} / V$ from equation (10). Also, because mostly finite wavevectors will be considered, the non-zero averages at $q=0$ often will be suppressed so that we have for fluctuations ' $\delta f_{\mathbf{q}}=f_{\mathbf{q}}$ '.

Similarly, wavevector-dependent steady-state structure functions from equation (4) obey $S_{f_{\mathbf{k}} g_{\mathbf{q}}}=N S_{f g ; \mathbf{q}} \delta_{\mathbf{k}, \mathbf{q}}$, where

$$
S_{f g ; \mathbf{q}}(\dot{\gamma})=\frac{1}{N}\left\langle\delta f_{\mathbf{q}}^{*} \delta g_{\mathbf{q}}\right\rangle^{(\dot{\gamma})} .
$$

The familiar structure factor built with density fluctuations shall be abbreviated by $S_{\mathbf{q}}(\dot{\gamma})=$ $\frac{1}{N}\left\langle\varrho_{\mathbf{q}}^{*} \varrho_{\mathbf{q}}\right\rangle^{(\dot{\gamma})}$. While these findings are familiar from systems without shear, translational invariance of sheared systems takes a special form for the two-time correlation functions from equation (4). Because

$$
C_{f_{\mathbf{k}} g_{\mathbf{q}}}(t)=\mathrm{e}^{-\mathrm{i}(\mathbf{q} \cdot \kappa t+\mathbf{q}-\mathbf{k}) \cdot \mathbf{a}} C_{f_{\mathbf{k}} g_{\mathbf{q}}}(t),
$$

as follows from equation (21), a fluctuation with wavevector $\mathbf{q}$ is correlated with a fluctuation of $\mathbf{k}=\mathbf{q}(t)$ with the advected wavevector $\mathbf{q}(t)=\mathbf{q}+\mathbf{q} \cdot \boldsymbol{\kappa} t$ at the later time $t$; only then does the exponential in the last equation become unity. The advected wavevector's $y$-component increases with time as $q_{y}(t)=q_{y}+\dot{\gamma} t q_{x}$, corresponding to a decreasing wavelength, which the shear-advected fluctuation exhibits along the $y$-direction. Taking into account this time dependence of the wavelength of fluctuations, a correlation function characterized by a single wavevector can be defined, which resembles the equilibrium quantity: $C_{f_{\mathbf{k}} g_{\mathbf{q}}}(t)=N C_{f g ; \mathbf{q}}(t) \delta_{\mathbf{q}(t), \mathbf{k}}$ with:

$$
C_{f g ; \mathbf{q}}(t)=\frac{1}{N}\left\langle\delta f_{\mathbf{q}(t)}^{*} \mathrm{e}^{\Omega^{\dagger} t} \delta g_{\mathbf{q}}\right\rangle^{(\dot{\gamma})}, \quad \text { with } \mathbf{q}(t)=\mathbf{q}+\mathbf{q} \cdot \kappa t
$$


Picking out density fluctuations $\varrho_{\mathbf{q}}(t)$ again, the abbreviation $C_{\mathbf{q}}(t)=\frac{1}{N}\left\langle\varrho_{\mathbf{q}(t)}^{*} \mathrm{e}^{\Omega^{\dagger} t} \varrho_{\mathbf{q}}\right\rangle^{(\dot{\gamma})}$ for the intermediate scattering function under shear will be used. Similarly for the susceptibilities from equation (4), one finds $\chi_{f_{\mathbf{k}} g_{\mathbf{q}}}(t)=N \chi_{f g ; \mathbf{q}}(t) \delta_{\mathbf{q}(t), \mathbf{k}}$ with the result

$$
\chi_{f g ; \mathbf{q}}(t)=\frac{1}{N}\left\langle\sum_{i} \frac{\partial f_{\mathbf{q}(t)}^{*}}{\partial \mathbf{r}_{i}} \cdot \partial_{i} \mathrm{e}^{\Omega^{\dagger} t} g_{\mathbf{q}}\right\rangle^{(\dot{\gamma})},
$$

where the specialization to density variables shall be denoted by $\chi_{\mathbf{q}}(t)=\chi_{\varrho \varrho ; \mathbf{q}}(t)$.

While these expressions are easily formulated, they suffer from a lack of knowledge about $\Psi_{\mathrm{s}}$. Thus in the following a formal framework is developed within which to approximate $\Psi_{\mathrm{s}}$.

\section{Transient dynamics approach}

The following situation shall be studied: the system is in equilibrium at times $t \leqslant 0$, when instantaneously a constant shear rate $\dot{\gamma}$ is turned on:

$$
\Omega(\Gamma, t)= \begin{cases}\Omega_{\mathrm{e}}(\Gamma) & t \leqslant 0, \\ \Omega(\Gamma) & t>0,\end{cases}
$$

so that the distribution function at $t=0$ coincides with the equilibrium one, $\Psi(\Gamma, t=0)=$ $\Psi_{\mathrm{e}}(\Gamma)$. The solution of equations (1) and (26) is easily found for $t \geqslant 0$ :

$$
\Psi(\Gamma, t)=\mathrm{e}^{\Omega(\Gamma) t} \Psi_{\mathrm{e}}(\Gamma) .
$$

The switching-on of a real rheometer is supposed to influence the initial variation of $\Psi(t)$ only, which will be neglected in the following as the stationary state, presumably reached for $t \rightarrow \infty$, will be considered. Rewriting the exponential function,

$$
\mathrm{e}^{\Omega t}=1+\int_{0}^{t} \mathrm{~d} t^{\prime} \mathrm{e}^{\Omega t^{\prime}} \Omega
$$

leads, together with equations (5), (9), to the formal result for the steady-state distribution function (where physical units are restored, and the adjoint SO is introduced acting on the variables to be averaged with $\Psi_{\mathrm{s}}$ ):

$$
\Psi_{\mathrm{s}}(\Gamma)=\Psi_{\mathrm{e}}(\Gamma)+\frac{\dot{\gamma}}{k_{\mathrm{B}} T} \int_{0}^{\infty} \mathrm{d} t^{\prime} \Psi_{\mathrm{e}}(\Gamma) \sigma_{x y} \mathrm{e}^{\Omega^{\dagger}(\Gamma) t^{\prime}} .
$$

This simple result is central to our approach as it connects steady-state properties to time integrals formed with the shear-dependent dynamics. Knowledge about slow relaxation processes in the system can enter. Consequently, the steady-state averages from equation (22) are given by

$$
f(\dot{\gamma})=\left\langle f_{\mathbf{q}=\mathbf{0}}\right\rangle / V+\frac{\dot{\gamma}}{V} \int_{0}^{\infty} \mathrm{d} t^{\prime}\left\langle\sigma_{x y} \mathrm{e}^{\Omega^{\dagger} t^{\prime}} f_{\mathbf{q}=\mathbf{0}}\right\rangle
$$

while corresponding expressions hold for the structure functions from equation (23),

$$
S_{f g ; \mathbf{q}}(\dot{\gamma})=\left\langle\delta f_{\mathbf{q}}^{*} \delta g_{\mathbf{q}}\right\rangle / N+\frac{\dot{\gamma}}{N} \int_{0}^{\infty} \mathrm{d} t^{\prime}\left\langle\sigma_{x y} \mathrm{e}^{\Omega^{\dagger} t^{\prime}} \delta f_{\mathbf{q}}^{*} \delta g_{\mathbf{q}}\right\rangle
$$

the fluctuation functions from equation (24),

$$
C_{f g ; \mathbf{q}}(t)=\left\langle\delta f_{\mathbf{q}(t)}^{*} \mathrm{e}^{\Omega^{\dagger} t} \delta g_{\mathbf{q}}\right\rangle / N+\frac{\dot{\gamma}}{N} \int_{0}^{\infty} \mathrm{d} t^{\prime}\left\langle\sigma_{x y} \mathrm{e}^{\Omega^{\dagger} t^{\prime}} \delta f_{\mathbf{q}(t)}^{*} \mathrm{e}^{\Omega^{\dagger} t} \delta g_{\mathbf{q}}\right\rangle,
$$


and the susceptibilities from equation (25),

$$
\begin{aligned}
\chi_{f g ; \mathbf{q}}(t)=-\left\langle\delta f_{\mathbf{q}(t)}^{*} \Omega_{\mathrm{e}}^{\dagger} \mathrm{e}^{\Omega^{\dagger} t} \delta g_{\mathbf{q}}\right\rangle / N \\
\quad-\frac{\dot{\gamma}}{N} \int_{0}^{\infty} \mathrm{d} t^{\prime}\left\langle\sum_{i}\left(\mathbf{F}_{i}+\partial_{i}\right) \sigma_{x y} \mathrm{e}^{\Omega^{\dagger} t^{\prime}} \delta f_{\mathbf{q}(t)}^{*} \boldsymbol{\partial}_{i} \mathrm{e}^{\Omega^{\dagger} t} \delta g_{\mathbf{q}}\right\rangle .
\end{aligned}
$$

Note that the averages in equations (29), (32) can be performed with the known equilibrium distribution function. When studying the nonlinear rheology of simple fluids, transient correlation functions related to equation (29) were found useful in thermostatted simulations [15] and in mode-coupling approaches [14].

\subsection{Translational invariance reconsidered}

The time-dependent distribution function $\Psi(\Gamma, t)$ from equation (27) can be used to show that a translationally invariant equilibrium distribution function $\Psi_{\mathrm{e}}(\Gamma)$ leads to a translationally invariant steady-state distribution $\Psi_{\mathrm{s}}(\Gamma)$. To that end, as in section 2.2.3, $\Psi(\Gamma, t)$ is considered at the shifted positions, $\Gamma \rightarrow \Gamma^{\prime}$ with $\mathbf{r}_{i}^{\prime}=\mathbf{r}_{i}+\mathbf{a}$ for all $i$ :

$$
\Psi\left(\Gamma^{\prime}, t\right)=\mathrm{e}^{\Omega(\Gamma) t-\mathbf{P} \cdot \kappa \cdot \mathbf{a} t} \Psi_{\mathrm{e}}(\Gamma),
$$

where $\Psi_{\mathrm{e}}\left(\Gamma^{\prime}\right)=\Psi_{\mathrm{e}}(\Gamma)$ was used. The $\operatorname{SO} \Omega$ and the operator $\mathbf{P} \cdot \boldsymbol{\kappa} \cdot \mathbf{a}$ with $\mathbf{P}$ from equation (20) commute, because the shear rate tensor satisfies $\kappa \cdot \kappa=0$, and because the sum of all internal forces vanishes due to Newton's third law:

$$
\begin{aligned}
(\mathbf{P} \cdot \boldsymbol{\kappa} \cdot \mathbf{a}) \Omega & -\Omega(\mathbf{P} \cdot \boldsymbol{\kappa} \cdot \mathbf{a}) \\
= & \sum_{j}\left\{\left[\partial_{j}\left(\cdot \frac{\partial}{\partial \mathbf{r}_{j}}\left(\sum_{i} \frac{\partial U}{\partial \mathbf{r}_{i}} \cdot \boldsymbol{\kappa} \cdot \mathbf{a}\right)\right)\right]-\left[\left(\mathbf{a} \cdot \kappa^{\mathrm{T}} \cdot \boldsymbol{\kappa}^{\mathrm{T}} \cdot \partial_{j}\right)\right]\right\}=0 .
\end{aligned}
$$

Therefore, the Baker-Hausdorff theorem simplifies equation (33) to

$$
\begin{aligned}
\Psi\left(\Gamma^{\prime}, t\right) & =\mathrm{e}^{\Omega(\Gamma) t} \mathrm{e}^{-\mathbf{P} \cdot \kappa \cdot \mathbf{a} t} \Psi_{\mathrm{e}}(\Gamma) \\
& =\mathrm{e}^{\Omega(\Gamma) t} \mathrm{e}^{-\left(\sum_{i} \mathbf{F}_{i}\right) \cdot \kappa \cdot \mathbf{a} t} \Psi_{\mathrm{e}}(\Gamma) \\
& =\mathrm{e}^{\Omega(\Gamma) t} \Psi_{\mathrm{e}}(\Gamma),
\end{aligned}
$$

where the last equality again holds because the sum of all internal forces vanishes. Therefore,

$$
\Psi\left(\Gamma^{\prime}, t\right)=\Psi(\Gamma, t)
$$

holds, proving that the time-dependent and consequently the stationary distribution function $\Psi_{\mathrm{s}}(\Gamma)=\lim _{t \rightarrow \infty} \Psi(\Gamma, t)$ are translationally invariant even though the SO from equation (1) itself is not. This applies, at least, in cases without spontaneous symmetry breaking. Formally, the role of such symmetry breaking is to discard some parts of the steady-state distribution function and keep others (with the choice dependent on initial conditions). The distributions developed here discard nothing, and would therefore average over the disjoint symmetry-related states of a symmetry-broken system.

\subsection{Particle conservation}

For the present approach to be tenable, the time-dependent distribution function in equation (27) needs to approach $\Psi_{\mathrm{s}}$ at long times. Putting aside at first questions of non-ergodic glassy dynamics, the consequences of the conserved variables in the system need to be addressed. The particle number is the only conserved quantity, and its microscopic conservation law reads from equation (19):

$$
\partial_{t} \varrho_{\mathbf{q}}(t)=\Omega^{\dagger} \varrho_{\mathbf{q}}(t)=\mathrm{i} q j_{\mathbf{q}}^{\|}(t),
$$


with the particle flux given by the longitudinal element of the stress tensor (this holds generally for overdamped motion as the velocity is proportional to the force) and the drift flux, $j_{\mathbf{q}}^{\|}(t)=-\mathrm{i} q \sigma_{\mathbf{q}}^{\|}(t)-\mathrm{i} v_{\mathbf{q}}^{\kappa}(t)$, with

$$
\begin{aligned}
& \sigma_{\mathbf{q}}^{\|}(t)=-\mathrm{e}^{\Omega^{\dagger} t} \sum_{i}\left(1-\frac{\mathrm{i}}{q^{2}} \mathbf{q} \cdot \mathbf{F}_{i}\right) \mathrm{e}^{\mathrm{i} \mathbf{q} \cdot \mathbf{r}_{i}} \\
& v_{\mathbf{q}}^{\kappa}(t)=\mathrm{e}^{\Omega^{\dagger} t} \sum_{i} \frac{\mathrm{i} \mathbf{q} \cdot \boldsymbol{\kappa} \cdot \mathbf{r}_{i}}{q} \mathrm{e}^{\mathrm{i} \mathbf{q} \cdot \mathbf{r}_{i}} .
\end{aligned}
$$

To verify that particle number conservation does not prevent decay of the dynamics in equations (28), (32), the slow density fluctuations are eliminated using the equilibrium projection operator [2]:

$$
\left.P=\varrho_{\mathbf{q}}\right\rangle\left(N S_{q}\right)^{-1}\left\langle\varrho_{\mathbf{q}}^{*} \quad \text { where } P A=\varrho_{\mathbf{q}} \frac{\left\langle\varrho_{\mathbf{q}}^{*} A\right\rangle}{\left(N S_{q}\right)},\right.
$$

with complement $Q=1-P$, where sums over wavevectors $q$ are suppressed because of orthogonality. Here, the equilibrium static structure factor is abbreviated as $S_{q}=S_{\mathbf{q}}(\dot{\gamma}=$ $0)=\left\langle\varrho_{\mathbf{q}}^{*} \varrho_{\mathbf{q}}\right\rangle / N$ (it will appear repeatedly in the following), and idempotency $P^{2}=P$ is clear.

The correlation functions in equations (29), (32) can be abbreviated by $\left\langle\sigma_{x y} \mathrm{e}^{\Omega^{\dagger} t} X\right\rangle$, with $X=f_{\mathbf{0}}$ in equation (29), $X=\delta f_{\mathbf{q}}^{*} \delta g_{\mathbf{q}}$ in equation (30), and $X=\delta f_{\mathbf{q}\left(t^{\prime}\right)}^{*} \mathrm{e}^{\Omega^{\dagger} t^{\prime}} \delta g_{\mathbf{q}}$ in equation (31); employing the projector $P$ and recalling $\left\langle\sigma_{x y} \varrho_{\mathbf{q}}\right\rangle=0$, these become

$$
\left\langle\sigma_{x y} \mathrm{e}^{\Omega^{\dagger} t} X\right\rangle=\left\langle\sigma_{x y} Q \mathrm{e}^{\Omega^{\dagger} t} Q X\right\rangle+\left\langle\sigma_{x y} \mathrm{e}^{\Omega^{\dagger} t} \varrho_{\mathbf{q}}\right\rangle \frac{\left\langle\varrho_{\mathbf{q}}^{*} X\right\rangle}{N S_{q}} .
$$

In the first term on the right-hand side, already only fluctuations which are perpendicular to the hydrodynamic densities appear. The projector in equation (39) can be used to show the vanishing of the second term, where the slow hydrodynamic modes could enter. From equation (A.4) in the appendix $\left\langle\sigma_{x y} \mathrm{e}^{\Omega^{\dagger} t} \varrho_{\mathbf{q}}\right\rangle=0$ follows, as does the fact that reduced dynamics and full dynamics agree for the correlation functions needed in equations (29)-(32), namely:

$$
\left\langle\sigma_{x y} \mathrm{e}^{\Omega^{\dagger} t} X\right\rangle=\left\langle\sigma_{x y} Q \mathrm{e}^{\Omega^{\dagger} t} Q X\right\rangle=\left\langle\sigma_{x y} Q \mathrm{e}^{Q \Omega^{\dagger}} Q t Q X\right\rangle .
$$

The result is perhaps not surprising. The fact that density fluctuations are independent of the applied velocity field causes the dynamics leading to the changes in the stationary expectation values to be orthogonal to linear density fluctuations.

\subsection{Generalized Green-Kubo relations}

The result, equation (41), obtained from considering the conserved density fluctuations completes our derivation of generalized Green-Kubo relations. While the familiar GreenKubo relations of linear response theory connect transport coefficients to time integrals over projected fluxes [20], equations (29)-(32) enable one to calculate the steady-state properties of strongly sheared states far from equilibrium. Because of equation (41), the conserved density fluctuations do not contribute and the dynamics contains no hydrodynamically slow mode.

\section{Transient density fluctuations}

The problem of calculating steady-state averages is thus converted into one of first finding the transient dynamics after switching on the rheometer, and then integrating this in order to use equations (29)-(32). The transient density fluctuations will be important in this process 
(see [9]) and thus shall be simplified first. Because of the equivalence of the particles, the normalized transient collective intermediate scattering function can be written as

$$
\Phi_{\mathbf{q}}(t)=\frac{1}{N S_{q}}\left\langle\varrho_{\mathbf{q}(t)}^{*} \mathrm{e}^{\Omega^{\dagger} t} \varrho_{\mathbf{q}}\right\rangle=\frac{1}{S_{q}}\left\langle\varrho_{\mathbf{q}}^{\mathrm{s} *} \mathrm{e}^{-\mathrm{i} \mathbf{q} \cdot \kappa \cdot \mathbf{r}_{\mathrm{s}} t} \mathrm{e}^{\Omega^{\dagger} t} \varrho_{\mathbf{q}}\right\rangle
$$

where $\varrho_{\mathbf{q}}^{\mathrm{s}}=\mathrm{e}^{\mathrm{i} \mathbf{q} \cdot \mathbf{r}_{\mathrm{s}}}$ is the density of a single tagged particle, which is identical to the others. By this trick of singling out a particle, the motion of the surrounding particles due to the imprinted flow profile can be specified exactly in the time evolution described with one SO. By differentiating, one finds

$$
\partial_{t} \mathrm{e}^{-\mathrm{i} \mathbf{q} \cdot \kappa \cdot \mathbf{r}_{\mathrm{s}} t} \mathrm{e}^{\Omega^{\dagger} t}=\left(-\mathrm{iq} \cdot \boldsymbol{\kappa} \cdot \mathbf{r}_{\mathrm{s}}+\mathrm{e}^{-\mathrm{i} \mathbf{q} \cdot \kappa \cdot \mathbf{r}_{\mathrm{s}} t} \Omega^{\dagger} \mathrm{e}^{\mathrm{i} \mathbf{q} \cdot \kappa \cdot \mathbf{r}_{\mathrm{s}} t}\right) \mathrm{e}^{-\mathrm{i} \mathbf{q} \cdot \kappa \cdot \mathbf{r}_{\mathrm{s}} t} \mathrm{e}^{\Omega^{\dagger} t} .
$$

Integrating in time, the time evolution operator incorporating advection, in the case of density fluctuations, is found as

$$
\begin{aligned}
& \Phi_{\mathbf{q}}(t)=\frac{1}{S_{q}}\left\langle\varrho_{\mathbf{q}}^{\mathrm{s} *} \mathrm{e}_{+}^{\int_{0}^{t} \mathrm{~d} \tau \Omega_{\mathrm{s}}(\tau)} \varrho_{\mathbf{q}}\right\rangle, \\
& \Omega_{\mathrm{s}}(t)=-\mathrm{iq} \cdot \kappa \cdot \mathbf{r}_{\mathrm{s}}+\mathrm{e}^{-\mathrm{iq} \cdot \kappa \cdot \mathbf{r}_{\mathrm{s}} t} \Omega^{\dagger} \mathrm{e}^{\mathrm{i} \mathbf{q} \cdot \kappa \cdot \mathbf{r}_{\mathrm{s}} t},
\end{aligned}
$$

and $e_{+}$is the time-ordered exponential, where earlier times appear on the right. The time evolution operator can be worked out explicitly (but to little avail):

$$
\Omega_{\mathrm{s}}(t)=\Omega^{\dagger}-\mathrm{iq} \cdot \kappa \cdot \mathbf{r}_{\mathrm{s}}+\mathrm{iq} \cdot \kappa \cdot\left(2 \partial_{\mathrm{s}}+\mathbf{F}_{\mathrm{s}}\right) t-\mathbf{q} \cdot \kappa \cdot \kappa^{\mathrm{T}} \cdot \mathbf{q} t^{2},
$$

and because of shear-advection it is explicitly time dependent.

Projection operator manipulations [14] simplify the time-ordered exponentials, see the appendix, and from equation (A.8) follows an exact Zwanzig-Mori type equation of motion for the transient density correlators:

$$
\partial_{t} \Phi_{\mathbf{q}}(t)+\Gamma_{\mathbf{q}}(t) \Phi_{\mathbf{q}}(t)+\int_{0}^{t} \mathrm{~d} t^{\prime} M_{\mathbf{q}}\left(t, t^{\prime}\right) \Phi_{\mathbf{q}}\left(t^{\prime}\right)=0 .
$$

Here the static projector $\left.P_{\mathrm{s}}=\varrho_{\mathbf{q}}\right\rangle\left(1 / S_{q}\right)\left\langle\varrho_{\mathbf{q}}^{\mathrm{s} *}\right.$ was employed; because of the equivalence of the particles it again satisfies $P_{\mathrm{s}}^{2}=P_{\mathrm{s}}$. The time dependence of $\Omega_{\mathrm{s}}(t)$ leads to a slightly more general time dependence in equation (45) than is familiar in equilibrium. The initial decay rate' from equation (A.9) is

$$
\begin{aligned}
\Gamma_{\mathbf{q}}(t) & =-\frac{\left\langle\varrho_{\mathbf{q}}^{\mathrm{s} *} \Omega_{\mathbf{s}}(t) \varrho_{\mathbf{q}}\right\rangle}{S_{q}} \\
& =\frac{q^{2}+q_{x} q_{y} \dot{\gamma} t}{S_{q}}+\left(q_{x} q_{y} \dot{\gamma} t+q_{x}^{2} \dot{\gamma}^{2} t^{2}\right)-\frac{q_{x} q_{y}}{q S_{q}} \dot{\gamma} \frac{\partial S_{q}}{\partial q},
\end{aligned}
$$

which recovers the 'Taylor dispersion' familiar for non-interacting particles [2]; for noninteracting particles $M_{\mathbf{q}}\left(t, t^{\prime}\right)=0$ holds.

The memory function in equation (45) is given by

$$
\begin{aligned}
& M_{\mathbf{q}}\left(t, t^{\prime}\right)=-\left\langle A_{\mathbf{q}}^{\mathrm{s} *}(t) U_{\mathrm{s}}\left(t, t^{\prime}\right) B_{\mathbf{q}}^{\mathrm{s}}\left(t^{\prime}\right)\right\rangle / S_{q}, \quad \text { where } \\
& U_{\mathrm{s}}\left(t, t^{\prime}\right)=\mathrm{e}_{+}^{\int_{+}^{t} \mathrm{~d} \tau \Omega_{\mathrm{s}}(\tau) Q_{\mathrm{s}}},
\end{aligned}
$$

and where the generalized longitudinal stress tensor elements are

$$
\begin{aligned}
& \left\langle A_{\mathbf{q}}^{\mathrm{s} *}(t)=\left\langle\varrho_{\mathbf{q}}^{\mathrm{s} *} \Omega_{\mathrm{s}}(t) Q_{\mathrm{s}}\right.\right. \\
& \left.\left.B_{\mathbf{q}}^{\mathrm{s}}(t)\right\rangle=\Omega_{\mathrm{s}}(t) \varrho_{\mathbf{q}}\right\rangle,
\end{aligned}
$$


see equation (A.8). The memory function $M_{\mathbf{q}}\left(t, t^{\prime}\right)$ encodes the after-effects of the variables not treated explicitly in $\Phi_{\mathbf{q}}(t)$ that provide a bath for the density fluctuations.

In the context of mode-coupling theory, much depends on how this intractable object is approximated. There is ample evidence, for dense colloidal dispersions close to equilibrium, that a Markovian approximation for $M$ is quite insufficient [21]. Also evidence from careful dynamic light-scattering tests of mode-coupling theory close to equilibrium [22, 23] suggest that following Cichocki and Hess [24] a second projection step is required. We perform this step now; further discussion is deferred to section 4.1 below.

In the second projection operator step, the time evolution operator, equation (44), is formally [24, 25] decomposed as

$$
\begin{aligned}
\Omega_{\mathrm{s}}(t) & \left.=\Omega_{\mathrm{s}}^{i}(t)+\Omega_{\mathrm{s}}(t) \varrho_{\mathbf{q}}\right\rangle\left\langle\varrho_{\mathbf{q}}^{\mathrm{s} *} \Omega_{\mathrm{s}}(t) \varrho_{\mathbf{q}}\right\rangle^{-1}\left\langle\varrho_{\mathbf{q}}^{\mathrm{s} *} \Omega_{\mathrm{s}}(t)\right. \\
& =\Omega_{\mathrm{s}}^{i}(t)+\Omega_{\mathrm{s}}^{\mathrm{red}}(t),
\end{aligned}
$$

where the 'reducible' part of the SO couples the dynamics back to the generalized stress elements:

$$
\left.\Omega_{\mathrm{s}}^{\mathrm{red}}(t) Q_{\mathrm{s}}=-B_{\mathbf{q}}^{\mathrm{s}}(t)\right\rangle \frac{1}{S_{q} \Gamma_{\mathbf{q}}(t)}\left\langle A_{\mathbf{q}}^{\mathrm{s} *}(t) .\right.
$$

The importance of this separation of $\Omega_{\mathrm{s}}(t)$ lies in the possibility to introduce another memory function. While $M_{\mathbf{q}}\left(t, t^{\prime}\right)$ plays the role of a generalized diffusion kernel, the new memory function $m_{\mathbf{q}}\left(t, t^{\prime}\right)$ plays the role of a generalized friction kernel. As shown in the appendix, see equation (A.10), the original memory function can be rewritten using equation (49) as

$$
\begin{aligned}
M_{\mathbf{q}}\left(t, t^{\prime}\right) & +\Gamma_{\mathbf{q}}(t) m_{\mathbf{q}}\left(t, t^{\prime}\right) \Gamma_{\mathbf{q}}\left(t^{\prime}\right) \\
& +\Gamma_{\mathbf{q}}(t) \int_{t^{\prime}}^{t} \mathrm{~d} t^{\prime \prime} m_{\mathbf{q}}\left(t, t^{\prime \prime}\right) M_{\mathbf{q}}\left(t^{\prime \prime}, t^{\prime}\right)=0,
\end{aligned}
$$

where the new memory function is defined as

$$
m_{\mathbf{q}}\left(t, t^{\prime}\right)=\Gamma_{\mathbf{q}}^{-1}(t)\left\langle A_{\mathbf{q}}^{\mathrm{s} *}(t) U_{\mathrm{s}}^{i}\left(t, t^{\prime}\right) B_{\mathbf{q}}^{\mathrm{s}}\left(t^{\prime}\right)\right\rangle \Gamma_{\mathbf{q}}^{-1}\left(t^{\prime}\right) / S_{q} .
$$

Its time dependence is given by the 'irreducible' [24, 25] dynamics introduced in equation (49):

$$
U_{\mathrm{s}}^{i}\left(t, t^{\prime}\right)=\mathrm{e}_{+}^{\int_{t^{\prime}}^{t} \mathrm{~d} \tau \Omega_{\mathrm{s}}^{i}(\tau) Q_{\mathrm{s}}}
$$

From the theory of Volterra integral equations [26], see equation (A.11) in the appendix, it follows that the equation of motion, equation (45), can thus be rewritten as

$$
\partial_{t} \Phi_{\mathbf{q}}(t)+\Gamma_{\mathbf{q}}(t)\left\{\Phi_{\mathbf{q}}(t)+\int_{0}^{t} \mathrm{~d} t^{\prime} m_{\mathbf{q}}\left(t, t^{\prime}\right) \partial_{t^{\prime}} \Phi_{\mathbf{q}}\left(t^{\prime}\right)\right\}=0
$$

Equation (54), together with the definition of the memory function $m$ in equation (52), is the central new result of the approach to shear thinning introduced in [9] and is derived explicitly here for the first time. Together with the generalized Green-Kubo relations of equations (29)(32), it will be the starting point for factorizations building on the insights of mode-coupling theory [10] into the dynamics of quiescent colloidal dispersions.

\subsection{Discussion of the memory functions $M$ and $m$}

The equations of motion containing the two memory functions differ because of the CichockiHess projection step in equation (49). The different contents of equations (45), (54) can 
be seen from performing a Markovian approximation in the two memory functions. Then, equation (45) becomes

$$
\partial_{t} \Phi_{\mathbf{q}}(t)+\left[\Gamma_{\mathbf{q}}(t)+\int_{0}^{t} \mathrm{~d} t^{\prime} M_{\mathbf{q}}\left(t, t^{\prime}\right)\right] \Phi_{\mathbf{q}}(t)=0,
$$

where the renormalization of the decay rate $\Gamma$ can be expected to be negative (without shear this can be shown rigorously, and is connected to equation (8)). In order to describe a slowing down of the dynamics (namely a small effective decay rate) thus a near cancellation of the two terms in the square bracket in equation (55) is required. Approximations will need to be subtle to recover this near cancellation.

On the other hand, equation (54) becomes, upon performing a Markovian approximation,

$$
\partial_{t} \Phi_{\mathbf{q}}(t)+\left[\Gamma_{\mathbf{q}}(t) /\left(1+\Gamma_{\mathbf{q}}(t) \int_{0}^{t} \mathrm{~d} t^{\prime} m_{\mathbf{q}}\left(t, t^{\prime}\right)\right)\right] \Phi_{\mathbf{q}}(t)=0,
$$

where the renormalization of the decay rate now describes a suppression of the dynamics (slowing down) as the memory contribution can be expected to be positive (again without shear rate this can be shown rigorously), and large. Any approximation yielding a large memory integral (as expected close to equilibrium in dense dispersions), thus can reasonably describe slowing down using equation (54) without running the risk to predict an unstable system, namely negative decay rates.

\subsection{Neutral or vorticity direction}

In the vorticity direction, $\mathbf{q}=q \hat{\mathbf{z}}$, perpendicular to the impressed solvent flow and its gradient, the equations (52), (54) simplify to almost the known ones from the standard Zwanzig-Mori approach. The equation of motion becomes

$$
\partial_{t} \Phi_{q \hat{\mathbf{z}}}(t)+\frac{q^{2}}{S_{q}}\left\{\Phi_{q \hat{\mathbf{z}}}(t)+\int_{0}^{t} \mathrm{~d} t^{\prime} m_{q \hat{\mathbf{z}}}\left(t, t^{\prime}\right) \partial_{t^{\prime}} \Phi_{q \hat{\mathbf{z}}}\left(t^{\prime}\right)\right\}=0,
$$

with the simpler expression of the memory function:

$$
\begin{gathered}
m_{q \hat{\mathbf{z}}}\left(t, t^{\prime}\right)=\left\langle\left(\sigma_{q \hat{\mathbf{z}}}^{\| *}+V_{q \hat{\mathbf{z}}}^{\kappa *}\right) Q \mathrm{e}^{\Omega_{z}^{i}\left(t-t^{\prime}\right)} \sigma_{q \hat{\mathbf{z}}}^{\|}\right\rangle S_{q} / N, \quad \text { where } \\
V_{q \hat{\mathbf{z}}}^{\kappa}=\sum_{j l} \mathrm{e}^{\mathrm{i} q z_{l}} \frac{\mathbf{r}_{j} \cdot \boldsymbol{\kappa}^{\mathrm{T}} \cdot \mathbf{F}_{j}}{q^{2}} Q .
\end{gathered}
$$

The stress tensor $\sigma_{q}^{\|}$was defined in equation (38). To simplify $m$ the equivalence of the particles was used, replacing the single particle fluctuation $\varrho_{\mathbf{q}}^{\text {s }}$ by the collective one $\varrho_{\mathbf{q}} / N$ in all averages where the index $s$ appears only once. Importantly, shearing affects the vorticity direction not only via $V^{\kappa}$, but also via the reduced dynamics which couples all spatial directions and contains the shear rate $\dot{\gamma}$ in any order:

$$
\left.\Omega_{z}^{i}=\Omega^{\dagger} Q+\sigma_{q \hat{\mathbf{z}}}^{\|}\right\rangle \frac{1}{q^{2}}\left\langle\left(\sigma_{q \hat{\mathbf{z}}}^{\| *}+V_{q \hat{\mathbf{z}}}^{\kappa *}\right) Q .\right.
$$

Besides the reassurance that the formal manipulations recover almost standard results in the case where shearing affects the particle motion least, the resulting equation (58) for the memory function in the vorticity direction is noteworthy for two reasons. First, the stress-stress autocorrelation function $m$ calculated from equation (1) without shear arises from potential interactions and thus approaches a constant for vanishing wavevector, $m_{q}(t) \rightarrow m_{0}(t)<\infty$ for $q \rightarrow 0$ [21]. With shear, however, the result that $q^{2} m_{q \hat{\mathbf{z}}}\left(t, t^{\prime}\right) \rightarrow$ const for $q \rightarrow 0$ can be expected from equation (58), because the particles are forced by the flow field. Thus the 
hydrodynamic collective diffusion process will be affected. Second, the complicated time dependence which arises in the memory function $m_{\mathbf{q}}\left(t, t^{\prime}\right)$ in the $x$-and $y$-direction because of the advection of stress fluctuations with the imposed flow simplifies to a dependence solely on the time difference between stress fluctuations along the vorticity direction, $m_{q \hat{\mathbf{z}}}\left(t, t^{\prime}\right)=$ $m_{q \hat{\mathbf{z}}}\left(t-t^{\prime}\right)$.

\section{Discussion and outlook}

The derived generalized Green-Kubo relations of equations (29)-(32), and the equations of motion for the transient density correlators, equation (54), follow from the Smoluchowski equation for Brownian particles under uniform imposed shear as given in equation (1). From the Green-Kubo relations, general conclusions about linear response around the equilibrium state can be made by setting $\dot{\gamma}=0$ in the dynamics. The reduced dynamics in equation (41) contains no hydrodynamic components, because the density is the only conserved variable in Brownian systems. As long as the equilibrium fluctuations are ergodic and decay faster than $1 / t$ for long times, the leading change in any stationary variable is linear in shear rate $\dot{\gamma}$. Nonlinearities in $\dot{\gamma}$ in steady-state quantities will be largest for variables where the transient dynamics exhibits the slowest algebraic decay.

A central approximation of the approach is hidden in our postulate that the time-dependent solutions to the Smoluchowski equation approach the stationary solution at long times. Ageing effects [27] could prevent glassy quiescent states to follow the transient dynamics calculated above. Spatial symmetry breaking could lead to inhomogeneous states, such as 'shear-banded' ones. Only comparison with simulations and experiments can determine whether systems exist exhibiting the postulated properties.

A central difference from standard equilibrium Zwanzig-Mori equations for density fluctuations is the appearance of the time-dependent wavevector advection in the time-evolution operator $\Omega_{\mathrm{s}}(t)$ of equation (43). It arises because of the affine deformation of fluctuations and is an exact consequence of shear in the Smoluchowski equation (1). As found for simple liquids [16, 17], the mode-coupling approximations described in $[9,12,13]$ deduce from it that shear speeds up the structural relaxation and thus causes shear-thinning. The aspect that the stationary non-equilibrium state is characterized by a non-vanishing probability current, which is connected to the non-Hermitian nature of the Smoluchowski operator, enters our approach in the strategy to calculate the steady-state distribution function via integrating through the transient.

As discussed in section 4.1, the memory function $m_{\mathbf{q}}\left(t, t^{\prime}\right)$ from equation (54) and the equation of motion (52) appear reasonable starting points for approximations capturing the slow dynamics in driven (sheared) dense colloidal dispersions. Mode-coupling approximations had been suggested in [9], their universal contents had been discussed in [12], and their detailed presentation will be given in a future companion publication.

\section{Acknowledgments}

We thank M Ballauff, J Bergenholtz, Th Franosch, A Latz, K Kroy, and G Petekidis for discussions, and Th Franosch for a critical reading of the manuscript.

\section{Appendix A}

The appendix contains various more technical manipulations, which are used in the main text. 


\section{Appendix A.1}

The calculation of the linear response susceptibility in the stationary state starts from the change in the energy given in equation (7). The SO changes to $\Omega-\Delta \Omega(\Gamma, t)$, where

$$
\Delta \Omega(\Gamma, t)=\sum_{i} \partial_{i} \cdot\left(\frac{\partial}{\partial \mathbf{r}_{i}} f^{*}(\Gamma)\right) h_{\mathrm{e}}(t) .
$$

To linear order in the external field $h_{\mathrm{e}}$ the stationary distribution function changes to

$$
\Psi(\Gamma, t)=\Psi_{\mathrm{s}}(\Gamma)-\int_{-\infty}^{t} \mathrm{~d} t^{\prime} \mathrm{e}^{\Omega\left(t-t^{\prime}\right)} \Delta \Omega\left(\Gamma, t^{\prime}\right) \Psi_{\mathrm{s}}(\Gamma)+\mathcal{O}\left(h_{\mathrm{e}}^{2}\right),
$$

which leads to the shift of an arbitrary expectation value linear in the external field $h_{\mathrm{e}}$ given by

$$
\langle g\rangle^{\left(\dot{\gamma}, h_{\mathrm{e}}\right)}-\langle g\rangle^{(\dot{\gamma})}=-\int_{-\infty}^{t} \mathrm{~d} t^{\prime} g(\Gamma) \mathrm{e}^{\Omega\left(t-t^{\prime}\right)} \Delta \Omega\left(\Gamma t^{\prime}\right) \Psi_{\mathrm{s}}(\Gamma)+\mathcal{O}\left(h_{\mathrm{e}}^{2}\right) .
$$

A partial integration leads to equation (6) with the definition of the susceptibility in equation (4).

\section{Appendix A.2}

In order to show that conserved density fluctuations do not prevent the dynamics in equations (29)-(32) from relaxing, the following operator equality is useful, where the first line can be shown straightforwardly by differentiation:

$$
\begin{aligned}
\mathrm{e}^{\Omega^{\dagger} t} & =\mathrm{e}^{\Omega^{\dagger} Q t}+\int_{0}^{t} \mathrm{~d} t^{\prime} \mathrm{e}^{\Omega^{\dagger} t^{\prime}} \Omega^{\dagger} P \mathrm{e}^{\Omega^{\dagger} Q\left(t-t^{\prime}\right)} \\
& \left.=\mathrm{e}^{\Omega^{\dagger} Q t}+\frac{\mathrm{i} q}{N S_{q}} \int_{0}^{t} \mathrm{~d} t^{\prime} \mathrm{e}^{\Omega^{\dagger} t^{\prime}} j_{\mathbf{q}}^{\|}\right\rangle\left\langle\varrho_{\mathbf{q}}^{*} \mathrm{e}^{\Omega^{\dagger} Q\left(t-t^{\prime}\right)},\right.
\end{aligned}
$$

where $P$ is the projection operator from equation (39). Two conclusions can be drawn. First, because $\left\langle\sigma_{x y} \mathrm{e}^{\Omega^{\dagger} t} \varrho_{\mathbf{q}}\right\rangle$ in equation (40) by translational symmetry (see equation (21)) can be non-vanishing for $\mathbf{q}=0$ only, and there only the first term in equation (A.4) survives, it follows that

$$
\begin{aligned}
\left\langle\sigma_{x y} \mathrm{e}^{\Omega^{\dagger} t} \varrho_{\mathbf{q}}\right\rangle & =\left\langle\sigma_{x y} \mathrm{e}^{\Omega^{\dagger} Q t} \varrho_{\mathbf{q}}\right\rangle \delta_{\mathbf{q}, \mathbf{0}} \\
& =\left\langle\sigma_{x y} \varrho_{\mathbf{q}}\right\rangle \delta_{\mathbf{q}, \mathbf{0}}=0,
\end{aligned}
$$

as can be seen by expanding the exponential and using $Q \varrho_{\mathbf{q}}=0$. Consequently, equation (40) simplifies to

$$
\left\langle\sigma_{x y} \mathrm{e}^{\Omega^{\dagger} t} X\right\rangle=\left\langle\sigma_{x y} Q \mathrm{e}^{\Omega^{\dagger} t} Q X\right\rangle
$$

Second, reduced dynamics and full dynamics agree for the correlation functions needed in equations (29)-(32), namely:

$$
\left\langle\sigma_{x y} Q \mathrm{e}^{\Omega^{\dagger} t} Q X\right\rangle=\left\langle\sigma_{x y} Q \mathrm{e}^{\Omega^{\dagger}} Q t Q X\right\rangle,
$$

again, because of the vanishing of the difference (arising from the second term on the last line in equation (A.4)) at $q=0$. This result leads to equation (41). 


\section{Appendix A.3}

In order to derive the equation of motion for transient density fluctuations, the time-ordered product in equation (43) is rewritten using the projection operator $Q_{\mathrm{s}}$, which is the complement (namely $1=P_{\mathrm{s}}+Q_{\mathrm{s}}$ ) to $P_{\mathrm{s}}$ defined below equation (45):

$$
\mathrm{e}_{+}^{\int_{0}^{t} \mathrm{~d} \tau \Omega_{\mathrm{s}}(\tau)}=U_{\mathrm{s}}(t, 0)+\int_{0}^{t} \mathrm{~d} s U_{\mathrm{s}}(t, s) \Omega_{\mathrm{s}}(s) P_{\mathrm{s}} \mathrm{e}_{+}^{\int_{0}^{\mathrm{s}} \mathrm{d} \tau \Omega_{\mathrm{s}}(\tau)},
$$

with the abbreviation $U_{\mathrm{s}}\left(t, t^{\prime}\right)$ from equation (47). Equality can be shown by differentiation:

$$
\begin{aligned}
\partial_{t} \mathrm{e}_{+}^{\int_{+}^{t} \mathrm{~d} \tau \Omega_{\mathrm{s}}(\tau)}= & \Omega_{\mathrm{s}}(t) Q_{\mathrm{s}} U_{\mathrm{s}}(t, 0)+\Omega_{\mathrm{s}}(t) P_{\mathrm{s}} \mathrm{e}_{+}^{\int_{0}^{t} \mathrm{~d} \tau \Omega_{\mathrm{s}}(\tau)} \\
& +\int_{0}^{t} \mathrm{~d} s \Omega_{\mathrm{s}}(t) Q_{\mathrm{s}} U_{\mathrm{s}}(t, s) \Omega_{\mathrm{s}}(s) P_{\mathrm{s}} \mathrm{e}_{+}^{\int_{0}^{\mathrm{s}} \mathrm{d} \tau \Omega_{\mathrm{s}}(\tau)},
\end{aligned}
$$

where $\partial_{t} U_{\mathrm{s}}\left(t, t^{\prime}\right)=\Omega_{\mathrm{s}}(t) Q_{\mathrm{s}} U_{\mathrm{s}}\left(t, t^{\prime}\right)$ was used; regrouping on the right-hand side shows that $\left(\partial_{t}-\Omega_{\mathrm{s}}(t) Q_{\mathrm{s}}\right) \eta(t)=\Omega_{\mathrm{s}}(t) P_{\mathrm{s}} \exp _{+} \int_{0}^{t} \mathrm{~d} \tau \Omega_{\mathrm{s}}(\tau)$, where $\eta(t)$ abbreviates either the left- or right-hand side of equation (A.6). Yet, equation (A.7) turns out more useful when sandwiched between density fluctuations:

$$
\begin{aligned}
\partial_{t} \Phi_{\mathbf{q}}(t) S_{q}= & \left\langle\varrho_{\mathbf{q}}^{\mathrm{s} *} \partial_{t} \mathrm{e}_{+}^{\int_{0}^{t} \mathrm{~d} \tau \Omega_{\mathrm{s}}(\tau)} \varrho_{\mathbf{q}}\right\rangle=\frac{\left\langle\varrho_{\mathbf{q}}^{\mathrm{s} *} \Omega_{\mathrm{s}}(t) \varrho_{\mathbf{q}}\right\rangle}{S_{q}}\left\langle\varrho_{\mathbf{q}}^{\mathrm{s} *} \mathrm{e}_{+}^{\int_{0}^{t} \mathrm{~d} \tau \Omega_{\mathrm{s}}(\tau)} \varrho_{\mathbf{q}}\right\rangle \\
& +\int_{0}^{t} \mathrm{~d} s\left\langle\varrho_{\mathbf{q}}^{\mathrm{s} *} \Omega_{\mathrm{s}}(t) Q_{\mathrm{s}} U_{\mathrm{s}}(t, s) \Omega_{\mathrm{s}}(s) \varrho_{\mathbf{q}}\right\rangle \frac{1}{S_{q}}\left\langle\varrho_{\mathbf{q}}^{\mathrm{s} *} \mathrm{e}_{+}^{\int_{0}^{\mathrm{s}} \mathrm{d} \tau \Omega_{\mathrm{s}}(\tau)} \varrho_{\mathbf{q}}\right\rangle .
\end{aligned}
$$

Here, $Q_{\mathrm{s}} U_{\mathrm{s}}(t, 0) \varrho_{\mathbf{q}}=Q_{\mathrm{s}} \varrho_{\mathbf{q}}=0$ was used. Equation (45) follows and the definitions of the rate $\Gamma_{\mathbf{q}}(t)$ and the memory function $M_{\mathbf{q}}\left(t, t^{\prime}\right)$. The rate $\Gamma_{\mathbf{q}}(t)=-S_{q}\left\langle\varrho_{\mathbf{q}}^{\mathrm{s} *} \Omega_{\mathrm{s}}(t) \varrho_{\mathbf{q}}\right\rangle$ can easily be evaluated, using equation (44):

$$
\begin{aligned}
\left\langle\varrho_{\mathbf{q}}^{\mathrm{s} *} \Omega_{\mathrm{s}}(t) \varrho_{\mathbf{q}}\right\rangle= & \left\langle\varrho_{\mathbf{q}}^{\mathrm{s} *}\left(\Omega_{\mathrm{e}}-\mathbf{q} \cdot \kappa \cdot \boldsymbol{\kappa}^{\mathrm{T}} \cdot \mathbf{q} t^{2}\right) \varrho_{\mathbf{q}}\right\rangle \\
& +\mathrm{i} \mathbf{q} \cdot \kappa \cdot\left\langle\varrho_{\mathbf{q}}^{\mathrm{s} *}\left(2 \partial_{\mathrm{s}}+\mathbf{F}_{\mathrm{s}}\right) \varrho_{\mathbf{q}}\right\rangle+\sum_{i}\left\langle\varrho_{\mathbf{q}}^{\mathrm{s} *}\left(\mathbf{r}_{i} \cdot \boldsymbol{\kappa}^{\mathrm{T}} \cdot \partial_{i}-\mathbf{q} \cdot \kappa \cdot \mathbf{r}_{\mathrm{s}} \delta_{i, s}\right) \varrho_{\mathbf{q}}\right\rangle \\
= & -q^{2}-\mathbf{q} \cdot \kappa \cdot \kappa^{\mathrm{T}} \cdot \mathbf{q} t^{2} S_{q}-\mathbf{q} \cdot \kappa \cdot \mathbf{q} t\left(1+S_{q}\right) \\
& +\sum_{i} \mathrm{iq} \cdot \kappa \cdot\left\langle\varrho_{\mathbf{q}}^{\mathrm{s} *}\left(\mathbf{r}_{i}-\mathbf{r}_{\mathrm{s}}\right) \mathrm{e}^{\mathrm{i} \mathbf{q} \mathbf{r}_{i}}\right\rangle
\end{aligned}
$$

which leads to the stated result, because the last term in equation (A.9) becomes $\mathbf{q} \cdot \kappa \cdot \frac{\partial}{\partial \mathbf{q}} S_{q}$.

\section{Appendix A.4}

The decomposition of $\Omega_{\mathrm{s}}(t)$ in equation (49) leads to the differential equation for the reduced dynamics:

$$
\partial_{t} U_{\mathrm{s}}\left(t, t^{\prime}\right)=\Omega_{\mathrm{s}}(t) Q_{\mathrm{s}} U_{\mathrm{s}}\left(t, t^{\prime}\right)=\Omega_{\mathrm{s}}^{i}(t) Q_{\mathrm{s}} U_{\mathrm{s}}\left(t, t^{\prime}\right)+\Omega_{\mathrm{s}}^{\mathrm{red}}(t) Q_{\mathrm{s}} U_{\mathrm{s}}\left(t, t^{\prime}\right),
$$

which can be viewed as a differential equation with $\Omega_{\mathrm{s}}^{\mathrm{red}}(t) Q_{\mathrm{s}} U_{\mathrm{s}}\left(t, t^{\prime}\right)$ as inhomogeneity. It can be integrated to give

$\left.U_{\mathrm{s}}\left(t, t^{\prime}\right)=U_{\mathrm{s}}^{i}\left(t, t^{\prime}\right)-\int_{t^{\prime}}^{t} \mathrm{~d} t^{\prime \prime} U_{\mathrm{s}}^{i}\left(t, t^{\prime \prime}\right) B_{\mathbf{q}}^{\mathrm{s}}\left(t^{\prime \prime}\right)\right\rangle \frac{1}{S_{q}} \Gamma_{\mathbf{q}}^{-1}\left(t^{\prime \prime}\right)\left\langle A_{\mathbf{q}}^{\mathrm{s} *}\left(t^{\prime \prime}\right) U_{\mathrm{s}}\left(t^{\prime \prime}, t^{\prime}\right)\right.$,

where the explicit expression for the reducible part from equation (50) was used, and the 'irreducible' fast dynamics $U_{\mathrm{s}}^{i}\left(t, t^{\prime}\right)$ corresponds to the solution of the homogeneous equation. It is given in equation (53). Inserting the expression for $U_{\mathrm{s}}\left(t, t^{\prime}\right)$ into the definition of $M_{\mathbf{q}}\left(t, t^{\prime}\right)$ 
in equation (47) immediately gives equation (51) with the definition of the memory function equation (52). The equation of motion equation (45) can be viewed as a Volterra integral equation of second kind for $\Phi_{\mathbf{q}}(t)$, with kernel proportional to $M_{\mathbf{q}}\left(t, t^{\prime}\right)$ and $-\partial_{t} \Phi_{\mathbf{q}}(t) / \Gamma_{\mathbf{q}}(t)$ as inhomogeneity:

$$
\Phi_{\mathbf{q}}(t)+\int_{0}^{t} \mathrm{~d} t^{\prime} \frac{1}{\Gamma_{\mathbf{q}}(t)} M_{\mathbf{q}}\left(t, t^{\prime}\right) \Phi_{\mathbf{q}}\left(t^{\prime}\right)=-\frac{1}{\Gamma_{\mathbf{q}}(t)} \partial_{t} \Phi_{\mathbf{q}}(t) .
$$

The solution is given by

$$
\Phi_{\mathbf{q}}(t)=-\frac{1}{\Gamma_{\mathbf{q}}(t)} \partial_{t} \Phi_{\mathbf{q}}(t)-\int_{0}^{t} \mathrm{~d} t^{\prime} \tilde{m}_{\mathbf{q}}\left(t, t^{\prime}\right) \Gamma_{\mathbf{q}}\left(t^{\prime}\right) \frac{1}{\Gamma_{\mathbf{q}}\left(t^{\prime}\right)} \partial_{t^{\prime}} \Phi_{\mathbf{q}}\left(t^{\prime}\right),
$$

where the resolvent kernel $\tilde{m}_{\mathbf{q}}\left(t, t^{\prime}\right)$ satisfies the integral equation [26]:

$$
\begin{aligned}
\frac{1}{\Gamma_{\mathbf{q}}(t)} M_{\mathbf{q}}\left(t, t^{\prime}\right) & +\tilde{m}_{\mathbf{q}}\left(t, t^{\prime}\right) \Gamma_{\mathbf{q}}\left(t^{\prime}\right) \\
& +\int_{t^{\prime}}^{t} \mathrm{~d} t^{\prime \prime} \tilde{m}_{\mathbf{q}}\left(t, t^{\prime \prime}\right) \Gamma_{\mathbf{q}}\left(t^{\prime \prime}\right) \frac{1}{\Gamma_{\mathbf{q}}\left(t^{\prime \prime}\right)} M_{\mathbf{q}}\left(t^{\prime \prime}, t^{\prime}\right)=0 .
\end{aligned}
$$

By comparison of equations (A.13), (51), the memory function $m_{\mathbf{q}}\left(t, t^{\prime}\right)$ of equation (52) is identified to agree with the resolvent kernel entering in equation (A.12), $\tilde{m}_{\mathbf{q}}\left(t, t^{\prime}\right)=m_{\mathbf{q}}\left(t, t^{\prime}\right)$. Thus equation (A.12) actually is equivalent to equation (54) as was to be shown.

\section{References}

[1] Russel W B, Saville D A and Schowalter W R 1989 Colloidal Dispersions (New York: Cambridge University Press)

[2] Dhont J K G 1996 An Introduction to Dynamics of Colloids (Amsterdam: Elsevier Science)

[3] Risken H 1989 The Fokker-Planck Equation (Berlin: Springer)

[4] Bergenholtz J, Brady J F and Vicic M 2002 J. Fluid Mech. 456239

[5] Derks D, Wisman H, van Blaaderen A and Imhof A 2004 J. Phys.: Condens. Matter 16 S3917

[6] Petekidis G, Moussaid A and Pusey P N 2002 Phys. Rev. E 66051402

[7] Strating P 1999 Phys. Rev. E 592175

[8] Senff H, Richtering W, Norhausen C, Weiss W and Ballauff M 1999 Langmuir 15102

[9] Fuchs M and Cates M E 2002 Phys. Rev. Lett. 89248304

[10] Götze W 1991 Liquids, Freezing and Glass Transition ed J-P Hansen, D Levesque and J Zinn-Justin (Amsterdam: North-Holland) p 287

[11] Cates M E and Milner S T 1989 Phys. Rev. Lett. 621856

[12] Fuchs M and Cates M E 2003 Faraday Discuss. 123267

[13] Fuchs M and Cates M E 2002 J. Phys.: Condens. Matter 15 S401

[14] Kawasaki K and Gunton J D 1973 Phys. Rev. A 82048

[15] Morriss G P and Evans D J 1987 Phys. Rev. A 35792

[16] Miyazaki K and Reichman D R 2002 Phys. Rev. E $66050501(\mathrm{R})$

[17] Miyazaki K, Reichman D R and Yamamoto R 2004 Phys. Rev. E 70011501

[18] Szamel G 2004 Phys. Rev. Lett. 93178301

[19] McLennan J A 1988 Introduction to Non-equilibrium Statistical Mechanics (New York: Prentice-Hall)

[20] Forster D 1975 Hydrodynamic Fluctuations, Broken Symmetry, and Correlation Functions (Reading, MA: Benjamin)

[21] Pusey P N 1991 Liquids, Freezing and Glass Transition ed J-P Hansen, D Levesque and J Zinn-Justin (Amsterdam: North-Holland) p 763

[22] van Megen W and Underwood S M 1993 Phys. Rev. Lett. 702766

[23] van Megen W and Underwood S M 1994 Phys. Rev. E 494206

[24] Cichocki B and Hess W 1987 Physica A 141475

[25] Kawasaki K 1995 Physica A 21561

[26] Tricomi F G 1957 Integral Equations (New York: Interscience)

[27] Latz A 2002 J. Stat. Phys. 109607 